L'HOMME L'Homme

$200 \mid 2011$

Décrire, écrire

\title{
Retour sur archives
}

ou comment Minot s'est écrit

Back to the Archives, or How Minot was Written

\section{Françoise Zonabend}

\section{(2) OpenEdition \\ Journals}

Édition électronique

URL : http://journals.openedition.org/lhomme/22872

DOI : $10.4000 /$ /homme.22872

ISSN : 1953-8103

Éditeur

Éditions de l'EHESS

\section{Édition imprimée}

Date de publication : 21 novembre 2011

Pagination : 113-140

ISSN : 0439-4216

\section{Référence électronique}

Françoise Zonabend, «Retour sur archives », L'Homme [En ligne], 200 | 2011, mis en ligne le 09 novembre 2013, consulté le 30 avril 2019. URL : http://journals.openedition.org/lhomme/22872 ; DOI : 10.4000//homme.22872

Ce document a été généré automatiquement le 30 avril 2019

(c) École des hautes études en sciences sociales 


\title{
Retour sur archives
}

\author{
ou comment Minot s'est écrit \\ Back to the Archives, or How Minot was Written
}

\section{Françoise Zonabend}

Je remercie Marie-Claude Pingaud pour sa lecture attentive de ce texte.

1 UNE DOUZAINE de grands et lourds cartons de déménagement sur lesquels était inscrit au feutre noir «Minot», et qui étaient entassés dans un coin de la bibliothèque du Laboratoire d'anthropologie sociale, tel m'apparut, un jour de janvier 2001, le gisement de ce qu'on appela bientôt les "archives Minot $»^{1}$. Ces cartons contenaient des dossiers estampillés par thèmes, constitués de pages manuscrites ou imprimées, de rouleaux où défilaient des généalogies dessinées, de cahiers et de carnets remplis de notes, des fiches cartonnées rangées par items, des liasses de pages d'entretiens reconstitués ou décryptés et tant d'autres "papiers" à la teneur infiniment variée. Cet ensemble de cartons rassemblait tous les matériaux - ou presque - à partir desquels Tina Jolas, Marie-Claude Pingaud, Yvonne Verdier et moi-même avions fabriqué nos travaux sur Minot, travaux qui ont donné naissance à trois livres publiés, signés individuellement, et à plus de 500 pages d'articles écrits seule ou à plusieurs, soit plus de 1000 pages publiées, auxquelles s'est ajoutée une exposition photographique présentée à l'école du village puis au Musée national des Arts et Traditions populaires et dans diverses villes de France, ce dont témoigne un catalogue imprimé. Ces écritures imagées ou narratives de l'enquête furent complétées par de nombreuses communications orales présentées, par l'une ou l'autre, dans des séminaires ou des colloques universitaires. C'est dire la quantité et la variété des expressions empruntées pour rendre compte de cette enquête. Dès lors, faut-il encore s'interroger sur une entreprise amplement publiée et tout aussi, du reste, amplement commentée et critiquée ? Faut-il garder ces encombrants cartons, cataloguer, classer, en un mot archiver le contenu disparate de tous ces dossiers? De quoi peut bien être porteuse l'« archive » ainsi récupérée que n'ont pas révélé les travaux édités, les images montrées ou les exposés oraux présentés? 
Les “Dames" de Minot sur les marches de l'école primaire, en juillet 1972

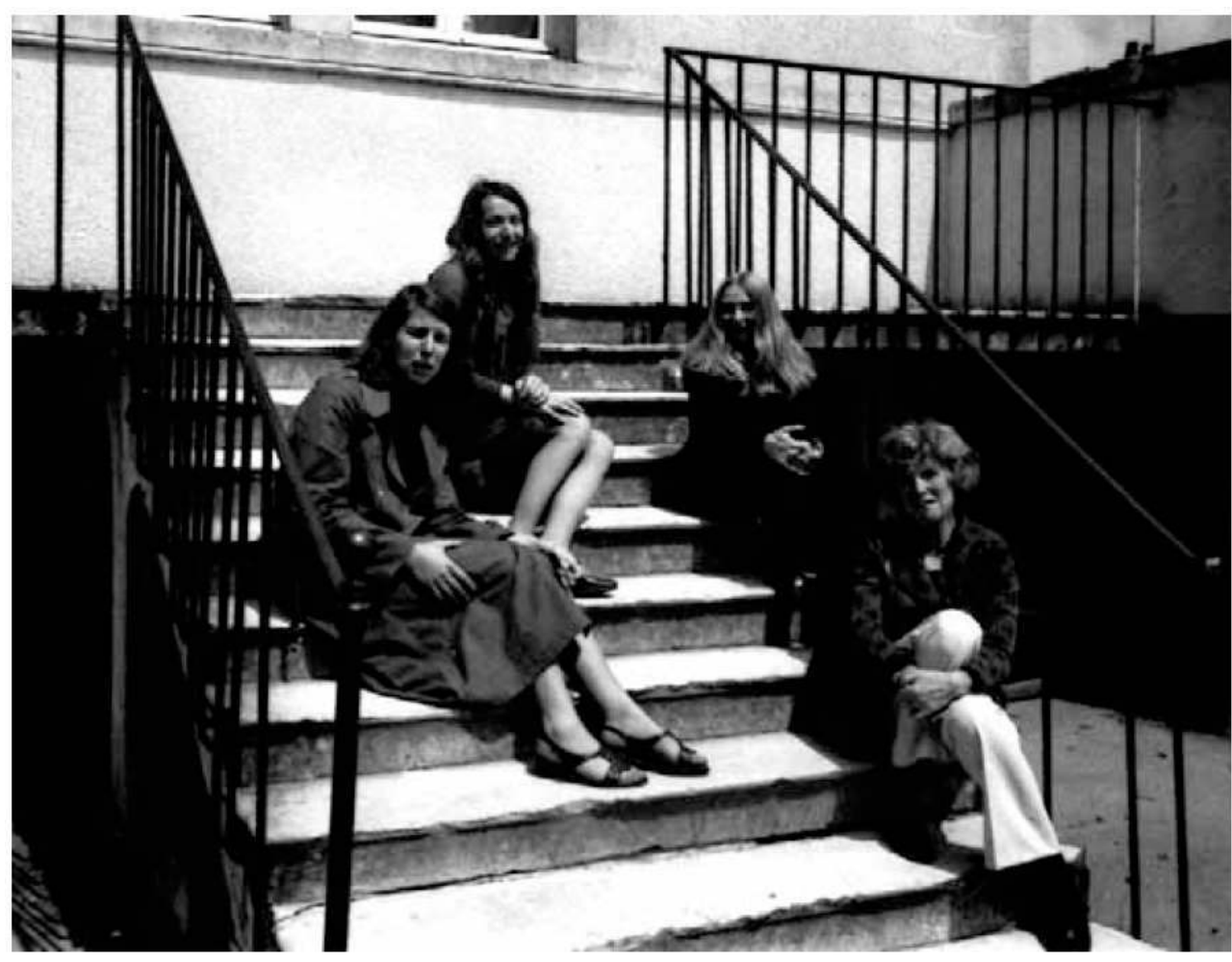

Cl. André Zonabend, archives du Laboratoire d'anthropologie sociale.

Des membres de l'équipe au travail dans l'espace des Human Relations Area Files, en 1984

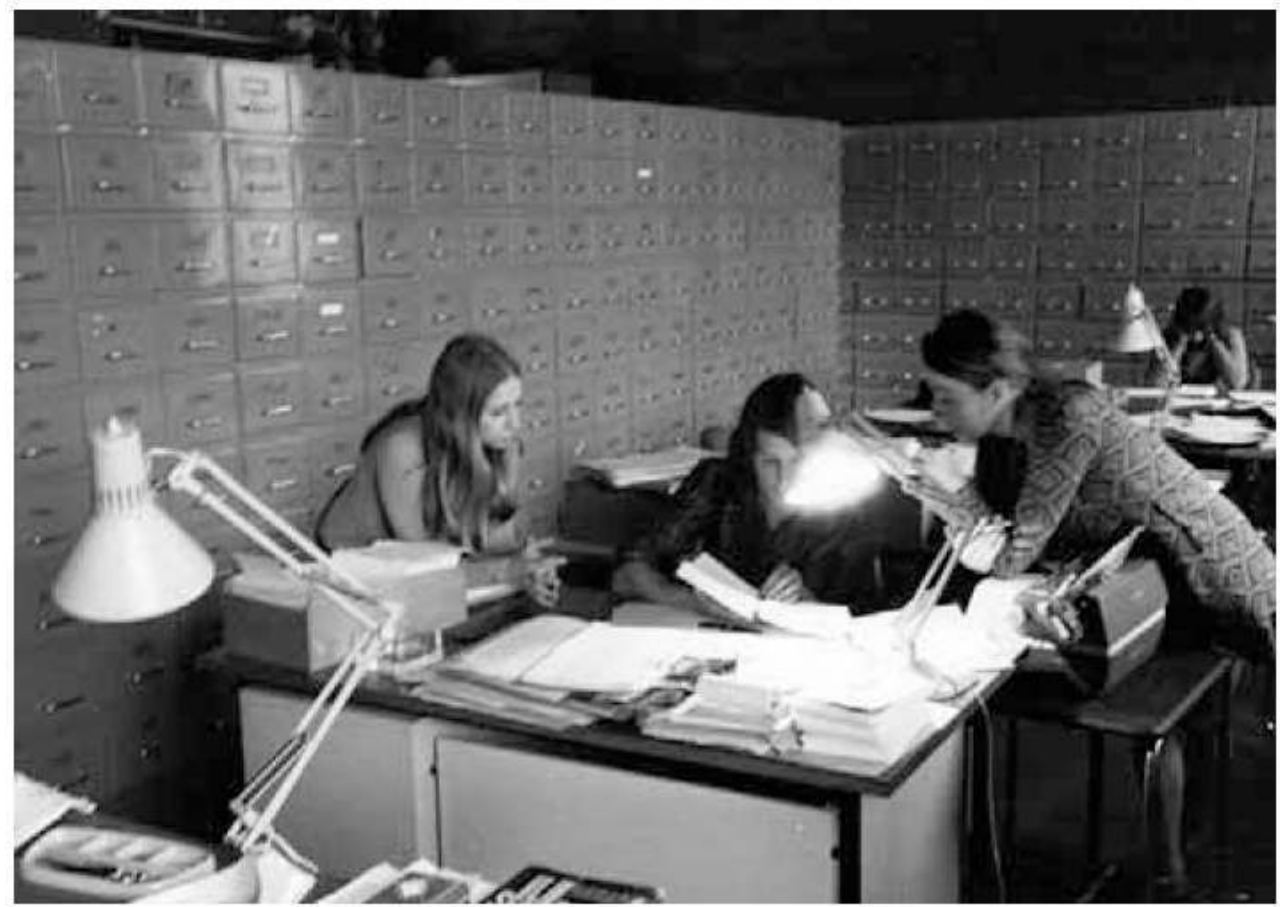

Cl. André Zonabend, archives du Laboratoire d'anthropologie sociale. 
$2 \mathrm{Au}$ demeurant, quand cette interrogation sur les «archives de Minot» a-t-elle commencé? Comment s'écrit l'histoire du surgissement de ces cartons étiquetés «Minot»? Pourquoi en parler, les évoquer maintenant, alors que plus de trente ans se sont écoulés depuis la parution de ces publications et que nous pensions en avoir fini avec Minot? Surtout que, pendant ce laps de temps, intervinrent divers événements qui nous éloignèrent de plus en plus de ce lieu et des travaux qui y furent menés. Il y eut d'abord l'épisode du procès (voir infra) qui nous persuada que tout retour à Minot était impossible. Puis, advint la mort accidentelle d'Yvonne Verdier' ${ }^{2}$, en août 1989, qui fit resurgir pour les autres membres du groupe le «fil rouge » des souvenirs de l'enquête ${ }^{3}$. Survint encore, en 1990, la demande de la Mission du patrimoine ethnologique de réunir en un seul ouvrage l'ensemble des articles parus sur Minot $^{4}$. Cette entreprise nous rassembla, nous les trois restantes, pour la dernière fois, afin de rédiger une présentation collective de nos travaux dans laquelle nous effleurâmes les façons dont nous avions travaillé sur le terrain. Puis, en septembre 1999, ce fut Tina Jolas qui décéda. L'aventure «Minot» était, semble-t-il, bien terminée et les dossiers de travail que nous avions constitués furent définitivement remisés dans un coin de mon bureau.

\section{Archiver?}

3 Pourtant j'aurais dû me rappeler que, lorsqu'en 1985 le Laboratoire d'anthropologie sociale quitta la place Marcelin-Berthelot pour emménager dans les locaux de l'ancienne École polytechnique rénovée, et que je dus faire un tri dans mes dossiers, je décidai d'entasser ceux de la Recherche coopérative sur programme (RCP Châtillonnais du CNRS) dans de grands cartons de déménagement sur lesquels j'inscrivis au feutre noir " Minot ». Ils furent alors déposés dans une des caves du Collège de France où je les oubliai. Ne restèrent, bien en évidence sur les étagères de mon nouveau bureau, que quelques objets, sortes d'indices mnémotechniques qui, lorsque je les contemplais, ravivaient en moi le temps et les incidents de l'enquête sur le terrain : une taille $e^{5}$ frustre donnée par l'ancien boulanger dont on avait repéré la pareille sur une photographie prise en 1900, pendue au mur de la boutique de l'épicier de l'époque ; un écheveau de chanvre récolté il y a plus de cinquante ans dans une des dernières chènevières de Minot ; une fève, récupérée chez un antiquaire de la région, minuscule colombe de céramique qu'on glissait dans le gâteau de Pentecôte, un rituel, semble-t-il, spécifiquement bourguignon; enfin, le vieil appareil photo et les quelque cent photographies sur plaques de verre prises, entre 1900 et 1910, par Hyppolite Amiot, le menuisier du village, l'ensemble nous fut donné par le dernier forgeron du village qui avait récupéré le tout in extremis sur un tas de fumier où on l'avait jeté6. Il me faut mentionner, cependant, que toutes les photographies recueillies auprès des habitants de Minot ou prises par nous-même furent, dès la fin de l'enquête de terrain, déposées à la photothèque de la bibliothèque du Laboratoire d'anthropologie sociale où elles sont toujours. Ces photographies ne subirent donc pas le même sort que les autres «papiers» de l'enquête. Toutefois, nous le verrons, elles nous furent de précieux auxiliaires pour forger des liens avec nos informateurs ou étoffer nos entretiens et, à cet égard, elles constituèrent de véritables instruments et documents de travail et auraient dû être classées avec les autres dossiers de l'enquête. Mais, d'emblée, nous avons eu le sentiment que le millier de photographies que nous avions rassemblées, et qui illustrent près d'un siècle de la vie du village - de 1880 à 1975 -, représentaient les vraies et uniques archives de Minot, et, qu'en elles seules, reposait la trace mémorielle de nos travaux. 
Aussi demeurèrent-elles à notre portée au Laboratoire, vivantes, disponibles en quelque sorte pour illustrer des ouvrages - les nôtres ${ }^{7}$ ou d'autres dont nous ne sommes pas signataires - ou des présentations orales de l'enquête. Pourquoi ces «images» et ces quelques « objets » n'ont-ils pas connu le même sort que les autres matériaux sur Minot? Pourquoi les avoir mis ailleurs que dans ces dossiers, avec ces fiches, ces feuillets couverts de nos écritures et autres documents rassemblés en un arrangement irraisonné dans des cartons, puis déposés dans la cave attribuée au Laboratoire? Il faut comprendre qu'à l'époque, lorsqu'il fallut entreposer ces cartons, pas un instant nous ne fûmes effleurées par l'idée qu'il s'agissait là d'archives, au sens classique du terme, et que nous devions les classer, les ranger et les laisser à la disposition de la communauté scientifique ${ }^{8}$. Aussi seule la partie iconographique de notre documentation fut-elle traitée comme d'authentiques archives. Quant aux dossiers "papiers ", leur conservation fut laissée au hasard et nous ne nous sommes guère préoccupées de leur destin. Il aura fallu que le Collège de France cherchât à récupérer ses caves pour que ces cartons ressurgissent et que je sois mise en demeure de m'en préoccuper.

4 Qu'en faire? Je m'apprêtai à tout jeter sur les conseils de Claude Lévi-Strauss (cf. épigraphe), lorsque, opportunément, la Maison des sciences de l'homme de Dijon décida de collecter les archives des études réalisées dans le cadre de la RCP du Châtillonnais dont Minot fit partie. Je décidai alors, en accord avec le Laboratoire d'anthropologie sociale et avec Marie-Claude Pingaud, d'envoyer tous ces cartons à Dijon. Toutefois, je réclamai qu'on dressât auparavant un inventaire précis de ce qu'ils contenaient.

Considérant alors la contextualisation de ces papiers inventoriés, je m'aperçus que le traitement dont ils devaient faire l'objet, lors de cette mise en archives, pouvait gauchir la réflexion épistémologique future qui risquait d'être menée à leur propos. Dans cet inventaire, en effet, chaque feuillet, chaque dossier, était nommément attribué à son inventeur à partir de l'écriture ou du thème traité, alors qu'aucun des documents n'était personnellement assigné ni signé. Aucun repère individuel ne marquait une quelconque appropriation ni appartenance de ces papiers, et ce simple établissement d'inventaire par ailleurs non exigé ${ }^{9}$ - occultait complètement l'aspect collectif des matériaux collectés. Dès lors, comment pallier ce biais sinon en témoignant aujourd'hui de ce que furent nos manières à toutes quatre de travailler sur le terrain, nos façons de constituer les unes pour les autres un corpus thématique, de puiser en commun aux mêmes sources de connaissance, toutes techniques d'enquêtes qui ne furent guère explicitées dans les articles ou ouvrages qui jalonnèrent ce travail.

6 Par ailleurs, je me rendis compte que, au fil de ma lecture de cet inventaire, les documents livrés ainsi en vrac reflétaient avant toute chose l'origine des savoirs que nous avions mobilisés, les types de contenus ethnographiques ou les partis pris scripturaux que nous avions utilisés pour fabriquer nos textes. Tous processus ou informations que nous avions omis de décrire dans les travaux publiés et qui, au demeurant, sont fort peu abordés dans les commentaires dont ces écrits firent l'objet où rien n'était dit - ou peu de chose - sur le genre d'écriture employé, sur l'accès et les formes d'utilisation des sources, ou encore sur les raisons des choix d'objets étudiés par chacune d'entre nous.

Ces dossiers, en effet, étaient des restes, vestiges de l'univers mémoriel dans lequel on évoluait à Minot: les archives locales publiques ou privées, les écrits populaires, littéraires ou savants portant sur la région, enfin la parole des habitants du village. Ces matériaux bruts «faisaient preuves », signes, des façons dont nous avions procédé pour mener nos travaux. Et, en parcourant l'inventaire de ces dossiers où l'on repère aussi bien 
des entretiens transcrits, des esquisses de schémas généalogiques, des listes de végétaux, des plans du cimetière ou de propriétés, en considérant le type de sources archivées (soit préexistantes soit produites par le chercheur), en remarquant les diverses formes de notations utilisées pour les dépouiller (liste, fiches, entretien oral restitué par écrit...), en repérant les points aveugles (absence de tout repère personnel ou de toute démarche réflexive), en faisant cela me sont alors revenus en mémoire les procédés intellectuels que nous avons suivis pour établir nos textes ainsi que la dimension émotionnelle qui les a soutenus sinon produits.

C'est dire la lecture ou l'exploration très particulière dont ces archives ont été l'objet de ma part. Elles devinrent les dispositifs mnémotechniques qui me permirent d'analyser et de percevoir ce que furent «nos » choix d'objet, «nos » écritures et, par l'effet de la distance temporelle qui s'était écoulée depuis leur confection, leur relecture avec un regard actuel, elles provoquèrent mon interrogation sur la fabrique des textes concernant Minot. D'autres décrypteurs de ces archives n'auraient certainement pas vu cela et, du reste, n'avaient aucune raison sérieuse de le faire : leurs enjeux sont ailleurs. Il n'y a que pour moi, lectrice impliquée, que ces archives se sont montrées révélatrices de nos pratiques, et représentaient des témoignages sur les manières dont nous nous y étions pris toutes quatre, sans nous consulter, pour écrire et décrire de façon harmonique "Minot». Il s'est agi, alors, de procéder à une expérience un peu particulière qui consistait en un retour, une revisite en quelque sorte dans des archives par une des auteures qui les a constituées et, dès lors, d'expliciter le mouvement de remémoration et de visualisation du travail de terrain dont elles ont été le déclencheur. En fait, c'est tout l'atelier de notre recherche qui me revenait en mémoire à la lecture de cet inventaire, et il m'a semblé possible de décrypter, à travers ces archives oubliées et redécouvertes, les façons dont « Minot s'est écrit ».

\section{Ethnographier}

7 Tout d'abord, remarquons que si ces archives se sont trouvées au Laboratoire d'anthropologie sociale et non au musée des Arts et Traditions populaires où sont déposées toutes les autres archives issues des travaux de la RCP du Châtillonnais, c'est que la recherche sur Minot s'inscrivit très vite, financièrement et scientifiquement, dans le cadre du Laboratoire et que les liens avec le dispositif central de la RCP furent très tôt rompus. De cette inscription institutionnelle découle le choix de nos méthodes de travail et de nos objets de recherche, lesquels relèvent avant tout de l'anthropologie classique, dite encore à l'époque "exotique", plutôt que de la conception muséographique, collecteuse et folkloriste de l'ethno-logie de la France poursuivie au musée des Arts et Traditions populaires. Partageant le même espace de travail, participant et présentant nos recherches aux mêmes séminaires - ceux de Claude Lévi-Strauss tout particulièrement -, nous avons d'emblée cherché à parler d'une même voix avec nos collègues « exotisants ", leur prouvant par nos pratiques de terrain et la construction de nos objets de recherche, que l'on pouvait étudier en ethnologue sa propre société, ce dont ils doutaient, persuadés qu'ils étaient encore, à ce moment-là, qu'une véritable ethnographie ne pouvait s'entreprendre qu'à bonne distance géographique et culturelle de soi. Au reste, de façon symptomatique, ils nous ont très vite surnommées les « Dames de Minot », en écho sans doute à l'appellation qu'on nous avait donnée au village et que 
nous leur avions rapportée, mi-amicale, mi-ironique, où nous étions devenues les « Dames de Paris ».

Nous inscrivant donc dans ce courant de l'anthropologie classique, nous sommes parties sur le terrain, certes pour " observer ", mais surtout pour " parler » avec les villageois de ce qui se passait là, en menant auprès d'eux des investigations directes comme cela se déroule dans toute enquête ethnographique. Pour ce faire, nous nous sommes installées dans la commune pour de fréquents et courts séjours, répartis dans l'année et au long de plusieurs années, afin d'établir avec les habitants une relation d'échange telle qu'ils acceptent notre inlassable curiosité au nom de notre intérêt pour leurs manières de vivre, aujourd'hui et autrefois. Cependant, il me faut dire que, mis à part quelques rituels familiaux, quelques fêtes calendaires ou des cérémonies communales collectives, nous avons peu " participé » à la vie domestique et sociale de nos informateurs, et assez peu partagé leur quotidien. D'ailleurs, ils ne le souhaitaient pas, préférant - nous l'avions toutes remarqué - s'entretenir avec nous chez eux, dans la salle commune, ou dehors, dans les jardins ou aux alentours du village, lors de rencontres convenues ou impromptues. Il est certain que ces façons de faire nous convenaient pour autant que nous avons très vite compris que les traits ou événements qu'il nous importait de discuter et les investigations que nous voulions mener avec eux, portaient sur un ensemble de faits jusqu'alors peu repérés dans l'ethno-logie de la France, tels le vocabulaire de la parenté, le partage des patrimoines entre lignées et entre fratries, les stratégies matrimoniales, le rôle de certaines femmes dans la construction de l'identité sexuée et sociale, la fabrique de la mémoire de l'histoire villageoise... Tous sujets qui, certes, ne sont pas que discours mais qui, dans la perspective de l'anthropologie sociale où nous les placions, étaient essentiellement de l'ordre du "parlé», du "récit» parce que, jusqu'alors, personne, dans les sociétés rurales européennes du moins, n'avait songé à les recueillir par écrit. Aussi, tout au long de cette enquête, avons-nous privilégié l'oral, l'échange discursif, l'entretien direct, recueillant leurs propos, leurs commentaires et prenant leurs mots au mot afin de pouvoir les restituer tels quels dans nos textes.

9 Toutefois, au début de la recherche, pour nourrir et enrichir ces nombreuses et interminables conversations que nous avions décidé de mener auprès des habitants, l'écrit - public ou privé - nous fut d'un grand secours. Ainsi nous sommes-nous d'abord appuyées sur les lectures des textes fondateurs de l'histoire des campagnes françaises. Celui, généraliste, de Marc Bloch ${ }^{10}$, mais aussi d'autres plus régionalistes, comme ceux de Gaston Roupnel ${ }^{11}$ et de Pierre de Saint-Jacob ${ }^{12}$. Nous avons également compulsé toute une littérature d'érudits locaux, d'historiens amateurs, d'écrivains régionaux ou hexagonaux, et, surtout, nous avons récolté et analysé des documents familiaux que nombre de nos informateurs nous fournirent obligeamment, puis consulté les multiples fonds archivistiques officiels que le village et la région recélaient à un point vertigineux. De sorte que nous avons dépouillé et utilisé les nombreux dossiers déposés à la mairie de la commune, au chef-lieu de canton ou au département: les Livres de Raison du dernier seigneur de Minot, les Cahiers de délibérations de la Justice seigneuriale du $\mathrm{Xv}^{\mathrm{e}}$ siècle à la Révolution, des liasses diverses contenant les actes de juridiction ou de règlement sur les bois, les foires, les fêtes, les listes nominatives des recensements depuis 1836, les états religieux et civils de 1690 à nos jours. Nous avons aussi étudié, avec application et grand intérêt, les six volumes manuscrits de L'Histoire de Minot, rédigés en 1913 par Georges Potey ${ }^{13}$, le dernier propriétaire du château, détenteur des archives seigneuriales, ainsi 
que la monographie composée en 1886 par Louis-Philippe Chaume ${ }^{14}$, instituteur au village, enfin celle confectionnée, dans les années 1930, par le dernier curé de la paroisse.

Toutes ces sources littéraires, savantes ou populaires, furent collectivement rassemblées, étudiées, consignées, mises en fiches et laissées à disposition de toutes. Ces dépouillements minutieux, commentés ou non, d'ouvrages savants et d'écrits officiels ou officieux constituent, du reste, une grande partie des «archives Minot », lesquelles sont donc composées de résumés de sources documentaires déjà existantes et élaborées. Nous avons, en quelque sorte, commencé par mettre en archive des archives.

11 Toutefois, au contraire de ce qui se pratiquait classiquement dans les travaux de sciences sociales de l'époque, et en particulier ceux des historiens et des sociologues, nous n'avons cherché à tirer de ces archives-archivées, si exhaustives qu'elles aient été, ni un inventaire des savoirs ou des techniques ni des éléments quantifiables ou des batteries d'indicateurs statistiquement jaugés, pas plus que nous n'avons tenté de retracer à partir d'elles l'histoire du village. Ces premières investigations scripturaires nous servirent avant tout à voir, mais surtout à entendre et à comprendre grâce à elles, ce que les gens de Minot avaient gardé en mémoire de leur propre histoire, de leurs façons de faire ou de leurs manières d'être ensemble. Tentant de "personnaliser » leur passé collectif et de retracer avec eux leur chronologie en débusquant la récurrence de leurs souvenirs, ou leur vision de leur espace naturel et social, nous cherchions à percevoir dans leur langage, leurs coutumes toujours pratiquées ou leurs comportements revendiqués, la résonance encore audible, vivante, de ces écrits anciens. Tous ces documents, en effet, usaient d'une langue, se servaient de mots et traitaient de faits dont nous allions « entendre », mais plus tard seulement, l'écho dans la parole des gens de Minot, où passé et présent se côtoyaient et se heurtaient sans cesse. De fait, c'est par l'expérience du terrain, par la connaissance acquise de façon directe auprès des villageois, que ces écrits archivés devinrent, pour nous, perceptibles, sensibles, utilisables.

$12 \mathrm{Au}$ départ, à la suggestion de la direction de la RCP, il avait été décidé que l'équipe du Laboratoire d'anthropologie sociale s'attacherait à mener à bien l'étude d'ensemble d'un des villages du Châtillonnais ${ }^{15}$. Nous étions donc parties de cette idée un peu confuse de monographie et étions convenues que celle-ci serait réalisée solidairement. Aussi, dès le début de la recherche, tous les dossiers de travail, les notes de terrain, les dépouillements d'archives de toutes sortes, nos lectures littéraires ou savantes, furent collectivement constitués et consultés par toutes. Il en alla de même des conversations ou des entretiens conduits individuellement ou de façon collégiale - parfois, à partir des guides élaborés ensemble, guides que je retrouve à profusion dans les archives et que, du reste, nous n'avions jamais avec nous pendant les entretiens !

13 Cette collecte profuse, tant verbale qu'éditoriale fut, dans un premier temps, mise en fiches dans ce que nous avons alors appelé notre fichier « Matières ». Au bout d'un certain temps, ce fichier comptait une demi-douzaine de longues boîtes en bois où toutes les informations étaient répertoriées, organisées par sujets selon les grands concepts de la discipline et où se mélangeaient indications «indigènes" et références "allogènes ». Pour ce faire, nous ramassions tous les faits en vrac, retenant, dans une ethnologie du fragment, des traits culturels s'inscrivant dans nos thèmes et consignés de façon aussi laconique que possible. Ce système de répertoire nous permit, du moins au début de l'enquête, de mieux communiquer les unes avec les autres : chacune prenant aisément connaissance de ce que les autres avaient indexé. Mais, nous comprîmes vite qu'en travaillant ainsi, nous rigidifions l'observation, nous procédions en quelque sorte déjà à 
une relecture aussi concise que brève des items étudiés. De plus, lors de nos entretiens, obsédées que nous étions par ce procédé de classement, nous en venions à poser des questions qui sollicitaient des réponses ponctuelles et thématiquement orientées, éludant, écartant toute information que nous estimions hors de nos champs conceptuels. Ce corpus de fiches rangées par matières se révéla donc, à l'usage, inopérant et nous dûmes y renoncer, constatant que nous nous trouvions devant une masse d'informations exsangues dont nous maîtrisions mal les correspondances. Surtout, il nous était apparu que mettre ainsi le village en fiches consistait finalement à le muséographier, à le placer par avance dans les vitrines d'un folklore, revisité certes, mais peu satisfaisant. Enfin, la forme monographique - synonyme de description tout à la fois singularisante et totalisante d'un groupe humain, menée selon un plan stéréotypé dans lequel nous devions nous inscrire - accentuait, nous sembla-t-il, cette impression d'émiettement et de cloisonnement de la culture villageoise que nous renvoyait notre fichier "Matières". Aussi son abandon s'accompagna-t-il du renoncement du projet de monographie commune, optant, chacune, pour des textes, collectifs ou individuels, illustrant divers aspects du groupe local tout en nous attachant, naturellement, à inscrire ces traits dans une description générale. Toutefois, malgré ce double abandon archivistique et éditorial et le choix de thèmes personnels à chaque chercheuse, la collectivisation des sources et des moyens documentaires demeura.

Désormais, nous procédâmes, chacune au bénéfice de toutes, à de multiples entretiens guidés par une volonté heuristique de compréhension, un souci de systématisation de certains traits sociaux, appuyés sur le récit de vie de la plupart des habitants de Minot. Cependant, nous avons pris soin de ne pas gommer les particularités de nos interlocuteurs, de ne pas réduire le vécu de chacun à l'expérience collective, ni de confondre la parole d'un seul avec la parole de tous. En sorte qu'avec cette façon de faire, l'autobiographie et la biographie trouvent leur place, mais une place spécifique qui permet de saisir l'individu aux prises avec les normes sociales, de découvrir les déterminations économiques, symboliques qui marquent une vie, pour autant que toute biographie ne prend son sens véritable que replacée dans le contexte social et historique dont elle est issue et où elle s'inscrit. C'est dire que le récit de vie isolé, privé du support de l'enquête ethnographique, apparaît comme une coquille vide. Éclairée par d'autres entretiens menés auprès d'autres interlocuteurs, relayée par l'histoire propre au groupe, la biographie devient alors un instrument de connaissance de la société. Ce sont ces récits de vie qui, principalement, se faufilent dans la trame des trois livres publiés sur Minot.

Nous eûmes aussi la bonne fortune d'entrer en possession de ces plaques photographiques évoquées plus haut, d'une remarquable qualité artistique et technique. Grâce à ces clichés que nous fîmes développer, surgit toute une génération d'hommes et de femmes saisis dans leurs tâches domestiques ou leur vie cérémonielle, et dont les plus âgés du village, au moment de l'enquête, avaient gardé le souvenir. Le déploiement dans les maisons de ces photographies anciennes fit sortir les boîtes et les albums serrés dans les armoires ${ }^{16}$. Ces clichés d'un passé plus récent relayèrent alors le fonds du menuisier et se raccordèrent aux photographies que nous prenions, ce qui nous permit d'illustrer, dans l'exposition photographique présentée au village en juillet 1972, la vie des gens de Minot pendant près d'un siècle. Autour de ces portraits et de ces images, anciennes et actuelles, se reformaient les cercles où se tissaient les destins individuels, où l'on " parlait famille", où l'on déclinait des histoires de vie, cercles au sein desquels nous étions désormais admises. 
Ce fut le moment charnière de l'enquête. Car, enfin, nous avions « entendu », dans ce qui nous était dit, cette langue juste, suggestive, forte, que les gens parlaient, s'agissant d'eux-mêmes et de ce qu'ils avaient à connaître autour d'eux. Parce qu'emprunte encore de particularismes dialectaux, leur langue était véhicule de sens, reflets de leurs pensées. Ce parler avait une expressivité propre, s'enracinant dans un lointain passé, et rendait compte d'une réalité dont la mémoire restait intacte et vivante. Beaux mots immémoriaux, restés incrustés dans la langue : saison, feu, affouage, dommage, pardon - «On tournait sur trois saisons"; " On partage l'affouage par feu »; "Il fallait veiller à ne pas laisser pâturer en dommage ou bien on allait au pardon». Verbes chargés d'une valeur transitive particulière subsumant toute une série de gestes et d'obligations : porter, courir, rendre "On porte à la noce» ; "On court conscrit»; " On se rend la main». Ou ces manipulations de mots et d'expressions qu'une simple torsion de la règle grammaticale rend déclinables et, dès lors, aptes à couvrir des domaines singuliers de la vie sociale : «vers" - qui peut venir à signifier le rapport d'apprentissage ; et "chez» - qui connote la famille non seulement en tant que maisonnée mais en tant que chaînon d'une lignée; de même, les multiples désinences du "champ les vaches", devenant selon les saisons et les parties en cause "Le champ la forêt ", "Le champ les moutons ", "Le champ les autres ", nous faisaient accéder aux emboîtements du terroir aussi bien qu'aux temps des enfances et des adolescences. Nous avons, dès lors, compris la vigueur du faire, notamment allié au mot coutume: «Faire la coutume» - qui s'applique aussi bien aux soins requis par le nouveau-né, aux façons de parer la mariée, aux gestes rituels que nécessite le défunt, qu'au découpage du sanglier et à la conduite des moutons sur les chaumes après la moisson. Enfin, nous avons saisi l'éthique des relations de parenté à travers ces renchaînements d'alliances que chacun ici favorisait, et qui se répétaient de génération en génération entre parentèles apparentées. À ce point, les entretiens devinrent de prolifiques conversations menées avec le seul souci $d u$ «laisser-parler». Pris en notes ou enregistrés, ces échanges verbaux, sollicités ou impromptus, étaient décryptés hâtivement le jour même, puis, de retour à Paris, patiemment reconstitués, rédigés et reproduits en plusieurs exemplaires. Poursuivi au long des mois d'enquête, repris de multiples fois sous des éclairages différents avec la même personne, l'ensemble de ces entretiens couvre des centaines de pages. On saisit bien, lorsqu'on les consulte aujourd'hui, combien ils ont été lus et relus, soulignés, annotés, utilisés et réutilisés par chacune d'entre nous pour servir ses propres préoccupations. D'où, certainement aussi, les répétitions d'un article à l'autre, d'une œuvre à l'autre, les mêmes données, les mêmes propos étant repris sous des éclairages distincts. Chacune a usé des mêmes mots, retenu les mêmes paroles des villageois pour illustrer, expliquer autrement un trait, un fait, écrivant, ainsi, une "ethnologie à multiples facettes». À partir d'une même phrase, d'un même mot, il y a toujours plusieurs révélations dont on peut discuter; plusieurs stratégies de compréhension sont toujours possibles. Si bien que nos écrits parfois se recoupent, souvent s'éclairent l'un par l'autre. En sorte que les textes publiés, même s'ils sont personnels, n'auraient sans doute pas pu être formulés sans cette collaboration intime, sans cette collectivisation des sources à partir de laquelle il est, en l'état actuel des archives, impossible de repérer l'ombre portée des textes publiés qui ont été tirés de celles-ci : aucun des ouvrages édités n'y est repérable puisqu'aucun manuscrit n'y figure ni qu'aucun dossier ne porte le titre d'un des écrits publiés. Aussi, dans cette collection de classeurs, ces fiches variées, ces notes griffonnées ou ces entretiens décryptés et annotés, la fabrique des œuvres s'efface au profit d'une image : celle du groupe au travail, décidé à puiser aux mêmes sources, de façon à en épuiser le sens. 
Cette "parole des enquêtés» fidèlement notée ou enregistrée constitue l'autre partie, quantitativement importante, des dossiers «Minot». Or, il m'apparaît aujourd'hui que ces entretiens sont les seuls «documents » incontestables que nous ayons produits et élaborés : là gisent, peut-être, les véritables archives de Minot !

Il est aussi important de souligner combien le mode choisi de présence au village, la durée de l'enquête et le groupe que nous formions, nous ont favorisées. Minot eut lieu à l'époque où nos institutions nous donnaient la possibilité de prendre du temps pour poursuivre nos travaux, aussi avons-nous pu mener un terrain morcelé et multiplié au long et au fil des années, et ce procédé d'enquête a joué un rôle fondamental non seulement dans la production des données mais, sans doute, dans leur analyse. Nous avons en effet pris le temps d'interroger et d'écouter les habitants du lieu: anciens résidents ou nouveaux venus, jeunes ou vieux, hommes ou femmes, agriculteurs ou artisans. Nous les avons tous rencontrés; avec presque tous, nous avons eu de longs et nombreux entretiens, avec certains les conversations furent multiples, pour ainsi dire sans fin. Ce fut en particulier le cas avec les membres les plus âgés du village, toujours disponibles et fins connaisseurs des mœurs et des pratiques locales, mais aussi avec le couple de jeunes instituteurs qui dirigeait, à l'époque, l'école de Minot. Quoiqu'ils fussent extérieurs à la région, avec ces derniers la convivialité fut d'emblée aisée, et leur participation à nos travaux ${ }^{17}$ fut enthousiaste et appréciable : ils acceptèrent, entre autres choses, de faire travailler leurs élèves au "Tableau du Temps » qui révélait, du point de vue des écoliers, le déroulement de la vie au village ${ }^{18}$, et nous permit de cerner de façon originale cette classe d'âge guère facile d'accès.

Et, parce que nous étions quatre, démultipliant l'investigation et revenant à intervalles réguliers, nos interlocuteurs étaient enclins, d'un entretien à l'autre, à la remémoration : sur place se créait une circulation de ces petits faits particuliers qui s'insèrent dans une chaîne d'informations dès lors cohérente, permettant de saisir une société dans sa complexe richesse. Les entretiens se transformèrent en récits que nous n'interrompions plus et qui gagnaient en densité grâce aux échanges qui avaient eu lieu en notre absence. Ils - et elles surtout - ne se lassaient pas d'évoquer le jeu des terres et des alliances, ce qui, au-delà de la parenté, conduisait aux manières de se conduire et de juger; ils se remémoraient les célébrations qui ordonnançaient le cycle de la vie; ils se rappelaient les alternances saisonnières entre finage et forêt qui rythmaient le temps au village; ils détaillaient les échanges qui se tissaient entre voisins de quartier. Nous comprîmes qu'à travers ces entretiens, ils nous racontaient leur ordre du monde.

\section{Écrire}

Quelques procédés scripturaux et éditoriaux constitutifs de notre anthropologie à Minot ont marqué les textes issus de cette recherche, accentuant, sans doute, sa singularité. Tout d'abord, il faut rappeler nos partis pris de publication. Contrairement aux normes retenues dans les autres RCP qui avaient eu lieu en France ${ }^{19}$, nous n'avons pas attendu la fin de l'enquête pour publier, sous forme d'articles rédigés au plus près du terrain, les résultats de nos recherches quitte à les ajuster, les amender, les réviser dans des publications ultérieures, au fur et à mesure de l'approfondissement de la recherche. Cette façon de faire s'explique certes par le réseau institutionnel et éditorial dont nous disposions au Laboratoire d'anthropologie sociale, et dans lequel s'inscrivaient ces travaux, mais aussi par le souci que nous avions de soumettre à la critique de nos 
collègues, nos capacités à « faire » une ethnologie chez soi. Il fallait qu'on s'impose, qu'on se positionne dans la discipline anthropologique en tant que chercheur at home.

Or, la lecture des articles qui jalonnent la période de l'enquête de terrain frappe par l'assurance naïve avec laquelle nous abordions la réalité profuse d'un village, comme si nous possédions une grille explicative qu'il suffisait d'appliquer. Ce ton catégorique correspond mal à ce que furent en vérité les tâtonnements des débuts, mais, dès les premiers articles, apparaissent en filigrane les théories et les méthodes auxquelles nous nous sommes référées. Les livres qui ont clos le terrain sont davantage argumentés et insérés dans la problématique globale de l'anthropologie sociale. Chacune choisissant la perspective qui lui convenait le mieux dans cette riche et complexe réalité sociale villageoise, nous avons $\mathrm{pu}$, dans ces espaces livresques, laisser libre cours au foisonnement de nos impressions, notations ou observations, cherchant avant tout à restituer aux gens de Minot toute la place que méritaient leurs gestes coutumiers, leurs histoires singulières, leurs figures tutélaires. Toutes façons d'écrire impossibles à adopter si nous avions dû nous couler dans le carcan rigide qu'imposait encore à l'époque l'écriture d'une monographie de facture classique, et cela explique aussi l'abandon du projet initial.

Qu'il s'agisse des articles ou des livres, tous ces travaux doivent beaucoup à Marcel Mauss dont l'influence se lit notamment dans l'article «Gens du finage, gens du bois ", où nous avons cherché à inscrire sur ce territoire contrasté les grandes alternances saisonnières selon les lieux, les modes de vie, les espaces de subsistance. Grâce à lui, nous avons porté une attention méticuleuse à l'ordre local, à son inscription sur l'espace et dans le temps. Mais, nous sommes surtout redevables à Claude Lévi-Strauss pour avoir souligné la portée significative des éléments disparates, la " prégnance du détail », nous incitant à recueillir minutieusement l'infime quotidien; aussi pour sa suggestion d'étendre les études de parenté aux sociétés complexes, de "faire parler les généalogies». Dans un premier temps du reste, nous nous étions attachées à reconstituer l'ensemble du réseau de parenté des villageois, espérant présomptueusement, grâce à l'état civil et paroissial, retrouver un grand ancêtre commun, ce à quoi, bien évidemment, nous avons dû renoncer. Le long rouleau de papier dénommé "Chrono-généalogie Girardot» répertorié dans les archives - constitue l'ultime indice de cette entreprise menée uniquement par dépouillement des états civils et paroissiaux. Devant le résultat esthétiquement réussi, mais scientifiquement creux, nous comprîmes, après avoir accompli ce long travail, combien la pratique ethnographique diffère du travail des historiens sur l'archive. Aussi est-ce par entretien direct, en face à face, que nous avons dressé les généalogies des gens de Minot qui nous ont alors enseigné à " parler famille ", et qui nous ont initiées à leurs patientes stratégies patrimoniales, comme à leurs façons de vivre avec leurs différents parents au sein de la communauté locale.

Alors nous avons écouté nos interlocuteurs nous parler de tout et de rien, du traditionnel, de l'usuel, de l'anodin, des pratiques et des faits qu'ils avaient retenus et dont ils ne parlaient qu'entre eux. Cette parole, nous l'avons retranscrite, fragmentée, désarticulée à des fins analytiques, dont nous avons supprimé, il est vrai, l'aspect "parlé », en consolidant leur message par l'écrit ${ }^{20}$.

$\mathrm{Au}$ terme de ce long terrain, chacune a puisé dans ces matériaux foisonnants mis en commun, choisissant la perspective qui lui convenait le mieux; chacune a développé son propos, tiré son fil: pour Tina Jolas, les rôles masculins, le calendrier des parcours cérémoniels et la solidarité des chasseurs; pour Marie-Claude Pingaud, l'espace, le 
paysage, l'usage des terres et la transmission des patrimoines; pour Yvonne Verdier, les protocoles complexes des repas rituels, les rôles symboliques et le destin physiologique des femmes ; pour moi, enfin, les relations de parenté, la mémoire, l'histoire, l'identité...

Il y aurait beaucoup à dire sur ces choix d'objets ; ils reflètent, à n'en pas douter, nos intérêts de l'époque, mais aussi nos affects personnels. Ils ont certainement à voir avec nos parcours biographiques ${ }^{21}$, avec nos curiosités intimement liées à nos passés, avec nos histoires personnelles, mais, dans tous les documents archivés ou dans les textes publiés, aucune annotation, aucun indice, ne vient étayer ces choix. En revanche, ce que ces dossiers ou ces textes reflètent, ce sont nos partis pris d'écriture.

Nous avions pris toutes quatre, sans nous concerter véritablement mais par souci d'assonance, le parti d'écrire dans un certain style ni trop savant ni trop simple. Une ethnographie en quelque sorte narrative, où l'écho des questions théoriques est plus implicite qu'explicite et où l'écriture est harmonieusement équilibrée entre le discours de nos informateurs et les analyses que ceux-ci nous suggéraient ou que nous découvrions, mais sans pour autant substituer nos propres mots aux leurs. Pour écrire ainsi, nous avons mobilisé, de façon plus ou moins appuyée selon l'une ou l'autre des chercheuses, certains procédés littéraires ou rhétoriques, nous évadant du village en ayant recours à des matériaux issus d'œuvres romanesques, poétiques ou picturales qui avaient, nous semble-t-il, à leur manière, saisi les caractères originaux de ces genres de vie. Ces façons d'écrire doivent beaucoup - du moins pour Yvonne Verdier et pour moi-même - aux procédés rédactionnels adoptés dès la confection de nos textes collectifs. Certes, écrire à deux ou à trois n'a pas été facile: il fallait que les autres acceptassent ce que l'une proposait comme analyse, comme idée, de façon à ce qu'on s'y reconnût toutes. Je me souviens : on s'ajustait les unes avec les autres, on arrivait à un texte, on intervenait, on se corrigeait, on s'écoutait, on réécrivait... Mais, celle qui souvent imprimait le rythme, le mot juste, c'était Tina, de qui nous avons beaucoup appris, Yvonne et moi. C'est à elle que nous devons quelques-uns de nos beaux titres d'articles : «Les verts anneaux acides: Ye demi-puppets that, by moonshine, do the green sour ringlets make... » (Shakespeare, The Tempest $, \mathrm{V}, 1$ ), ou cette majestueuse phrase de Marc Bloch, placardée à l'entrée de l'exposition photographique « ...Les tenaces ancêtres qui ont créé le blé, inventé le labourage et noué entre l'emblavure, la forêt et le paquis, une féconde alliance...». Aux connaissances littéraires de Tina, à son savoir-faire en écriture, le «style Minot » doit beaucoup.

Un autre trait commun à toutes quatre consistait en notre effacement personnel. Aucun des textes signés individuellement n'est écrit à la première personne, en nul endroit nous n'écrivons « je ». Pour autant, cela ne nous a pas empêchées, dans les ouvrages édités, de laisser libre cours au foisonnement de nos impressions, notations ou observations que nous suggérait notre travail de terrain. Est-ce le fait de la mise en commun des sources ou l'effet de l'écriture collective adoptée dans les premiers textes sur Minot qui nous interdirent les unes et les autres de poursuivre nos travaux sur un registre plus personnel? Je ne saurais le dire. Mais ce que l'on constate significativement c'est qu'aucune de ces archives ne relève de ce que René Loureau a nommé un «hors-texte » où le chercheur exprime sa subjectivité.

Nous sommes parties en quelque sorte de « leur » monde (le monde des gens de Minot) tel qu'ils le vivaient, s'en souvenaient, le décrivaient, pour l'interpréter ensuite dans une analyse anthropologique classique. Confrontées à la diversité des destins, à la variabilité des coutumes, à la multiplicité des usages, nous nous sommes efforcées de mettre au jour des permanences structurelles ou symboliques. Tout en nous appuyant sur l'observation 
de leurs pratiques quotidiennes, de leurs tactiques minuscules et sans cesse mouvantes, sur la révélation de leurs stratégies affirmées ou cachées, nous avons tenté de découvrir les logiques à l'œuvre et d'en dégager les archétypes qui donnent sens à une réalité sociale, la leur certes, mais, au-delà, celle des autres.

En effet, une question latente se posait à propos des travaux sur Minot: celle de leur représentativité. Quelle portée généralisante avaient nos «trouvailles » sociologiques ou symboliques, repérées dans cette communauté qui se présente comme une totalité ? Certes, ces villageois constituent une collectivité en soi, mais leur singularité ne les rend pas marginaux; on ne peut les abstraire de l'ensemble de la région où ils s'insèrent, ni des autres sociétés rurales européennes dont ils font partie intégrante. Au-delà des gens de Minot, de leur particularité et de leur spécificité, on peut déduire des régularités de comportements, des considérations d'ordre général, voire même des paradigmes sur les «styles de vie » des campagnes européennes ${ }^{22}$.

C'est ce que nous avons tenté de faire plus particulièrement avec l'étude d'un certain nombre de traits sociaux. Aussi avons-nous beaucoup insisté dans nos travaux sur les façons sociologiques, cérémonielles ou symboliques dont cette communauté découpait son espace cadastré où s'activaient en experts complémentaires les gens du finage et ceux du bois. Ce modèle, qui a une vaste portée en Europe occidentale, explique tout à la fois la structure architecturale et sociale du village, mais aussi les différences de traitement entre hommes et femmes, entre jeunes et vieux.

27 À propos de la parenté, nous avons pu restituer, par le recours aux méthodes classiques de l'anthropologie, les terminologies d'adresse et de désignation entre parents, permettant de la sorte des comparaisons entre systèmes de parenté européens et exotiques. De plus, en dressant avec nos informateurs leurs généalogies, nous avons mis en lumière ces mariages remarquables signalés ici par la périphrase : «les mariages se renchaînent ». Par cette formule, nos interlocuteurs désignaient les unions qui, dans une assez longue temporalité, renouvelaient les alliances entre mêmes lignées et que celles-ci avaient scrupuleusement gardées en mémoire. Ces échanges matrimoniaux, recherchés et répétés, qui renouaient les liens avec de lointains "apparentés ", étaient la preuve tangible qu'une règle insoupçonnée, intériorisée mais toujours suivie, ordonnait l'apparent désordre de leurs choix matrimoniaux. Grâce à ce constat, les recherches sur le rôle de la parenté au sein de nos sociétés occidentales à système complexe ont pris une autre dimension. Tout comme dans les sociétés à structures élémentaires, dans les communautés rurales, et, peut-être, dans d'autres groupes sociaux complexes, la parenté organise, arrange en tant que telle, le champ des alliances tout comme transitent, par elle, les biens symboliques ou patrimoniaux: «ici, sous le fouillis du divers, des régularités affleurent et suggèrent une invisible règle générale $»^{23}$.

28 En outre, nous voulions montrer comment le passé se perpétue ou resurgit dans le présent et comprendre pourquoi ce trait, associé à d'autres, donne continuité et permanence au groupe. Aussi, dans nos ouvrages, nous nous sommes attachées à saisir les façons dont une collectivité locale, sans cesse renouvelée au cours des temps, (re)construit son identité après chaque vague migratoire. Nous avons, dès lors, cherché à déchiffrer les relations que cette communauté entretient avec l'histoire globale, mais surtout avec son histoire, celle où les événements ne s'inscrivent pas forcément dans une perspective de changement et de progrès. Nous voulions prendre en compte, autrement, leurs manières de vivre "leur» temps ou de décoder, localement, les événements historiques. Il s'est agi, pour nous en quelque sorte, de tenter d'appréhender leurs 
conceptualisations locales de leur histoire et la diversité de leurs façons de mesurer et d'exprimer le temps. Nous ne recherchions pas une vérité, mais leur vérité, nous ne voulions pas recueillir leur façon de voir l'histoire, mais leur manière de dire leur propre histoire. En d'autres termes, nous avons été d'abord attentives au style exact de nos interlocuteurs qui sélectionnaient dans leur mémoire ce qu'ils voulaient dire ou désiraient se rappeler. Ce fut notre façon de conduire ce que d'autres ${ }^{24}$ ont appelé - mais sans vraiment le comprendre - une enquête d'« ethno- histoire » qui, de fait, rompait avec les études historiques classiques, mais s'ouvrait sur de nouvelles perspectives : sur le repérage des outils mnémotechniques grâce auxquels les groupes sociaux tissent leur temps, jouent de leur mémoire, perçoivent l'histoire et la font leur.

Lors de ces investigations, nous avons d'un commun accord privilégié le passé, ou plus exactement un passé en voie de transformation sous nos yeux, et cherché à décrire l'ultime ordonnancement des lieux, des familles et des mots, selon une figure communautaire encore réelle dans le temps de l'enquête, même si nous l'avions déjà vu revêtir des formes nouvelles liées à un espace qui s'ouvrait largement. Une période donc, qui va du début du siècle dernier au lendemain de la dernière guerre, suivant en cela le discours de nos informateurs qui privilégiaient ce qui était de l'ordre du passé «l'autrefois, l'avant »- comme axe de réflexion de la pensée : «temps qui est aussi celui d'une culture matérielle où la primauté est encore donnée aux éléments, l'eau, le feu $»^{25}$. Ce parti pris explique, peut-être, les lacunes de l'ensemble : sur le politique, les tensions de l'histoire récente, la hiérarchie des sexes, les nouveaux métiers, les intérêts des jeunes dans le présent, l'irruption de la modernité et beaucoup d'autres traits encore... Il est toutefois important de souligner que nombre de ces aspects étaient, à l'époque de l'enquête, traités par la sociologie rurale sur le terrain français. Il est vrai que cela n'excuse en rien les nombreux «manques» de notre ethnologie à Minot. Néanmoins, à leur propos, je n'insisterai que sur deux aspects qu'on nous a souvent reproché d'avoir éludés : l'un touche au fait que nous étions quatre «chercheuses » sur ce terrain et, par conséquent, a-t-on avancé, nos choix d'objets et nos écritures se seraient trouvés affectés par notre identité sexuée ; l'autre a trait au " politique » que nous avons traité de biais, voire esquivé dans nos recherches.

Certes, nous étions quatre femmes sur le terrain, mais nous ne pensons pas avoir écrit une anthropologie spécifiquement féminine. C'est pourtant ainsi que nos travaux furent souvent caractérisés ${ }^{26}$. Or, j'ai décrit plus haut le contexte disciplinaire dans lequel ceuxci se sont inscrits : c'était le lieu (si proche, trop "propre " peut-être) dans lequel se poursuivirent ces travaux qui faisait problème et non pas le fait que nous étions une équipe de femmes. Si nous avons eu, à Minot, une autre écoute et un autre regard que ceux traditionnellement portés sur le monde villageois français, cela s'explique avant tout par les raisons conjoncturelles évoquées plus haut. Pas plus féminine dans l'écriture que féministe dans notre vision des femmes - autre reproche qui nous fut fait ${ }^{27}$ à propos de nos textes sur Minot. Les mouvements féministes étaient encore balbutiants en France, à l'époque où nous travaillions au village, et nous n'étions guère, alors, partie prenante de leur problématique. Même si les unes et les autres, nous avons étudié en détail la condition des femmes au village, leur place dans l'organisation domestique, professionnelle ou cérémonielle, nous n'avons cherché ni à hiérarchiser ou stigmatiser les rapports de sexes, non plus qu'à montrer la subordination de l'un à l'autre ou les structures d'oppression entre hommes et femmes. Nos propos furent autres. Nous avons été surtout attentives à la fabrication des identités sexuées dans cette communauté villageoise, hier et aujourd'hui, sans insister, il est vrai, sur les rapports de domination 
entre les sexes. Il suffit, au demeurant pour en prendre acte, de relire les travaux d'Yvonne Verdier. En découvrant la place prépondérante de certaines femmes véritables "héroïnes culturelles" - lors des rites de passage les plus cruciaux qui scandent la vie de tout être humain, elle a révélé l'existence d'une véritable culture féminine incarnée chez ces " passeuse(s) aux gués du destin ${ }^{28}$. Des femmes qui assurent le strict contrôle des institutions par leur connaissance de la « coutume » à suivre et sont, dès lors, indispensables au bon agencement des corps et des ordres sociaux. L'étude de ces figures, comme d'autres, telle l'agricultrice dans les ouvrages de Marie-Claude Pingaud, la jardinière et la mercière dans La Mémoire longue, fut toujours menée dans leurs rapports complémentaires avec les hommes (bricolier, conscrit, chasseur ou meneur d'embuscade, tous dépistés par Tina Jolas dans ses articles) et ce, afin de poser la différence des sexes dans l'espace domestique ou villageois et donner sens à la place et aux rôles propres du masculin et du féminin dans les cycles individuels ou calendaires.

Une autre critique nous fut adressée ${ }^{29}$ : celle de n'avoir pas suffisamment discuté, avec les habitants de Minot, des forces politiques en présence au village. Pour ce faire, nous aurions dû débusquer leur amnésie sur la dernière guerre, dont l'évocation est totalement absente de nos travaux. Or, les événements qui s'y sont passés, les rôles clandestins ou officiels que certains chefs de famille ont alors endossés, fournissaient pourtant la clé de leurs choix politiques actuels. L'époque où se situe la recherche - la décennie 1970 explique sans doute le silence qui pesait encore sur ces années-là. Peu enclins à désigner les profiteurs et les dénonciateurs, les collaborateurs ou les maquisards, les gens de Minot ne nous en ont pas parlé spontanément. À leurs silences s'est ajoutée notre volonté, pour préserver notre cohésion, de ne pas faire intervenir nos histoires et blessures personnelles associées à ce passé proche. Sur ce point, notre évitement fut délibéré. Le temps n'était pas encore venu - ni pour eux ni pour nous - de parler sans réserve ni appréhension de ce qui s'était vraiment passé pendant l'Occupation.

Certainement, l'étonnante acuité du souvenir récurrent de croyances et de rites disparus depuis quelques décennies, la fraîcheur et la verdeur de témoignages sur la vie villageoise passée, les récits si denses et si minutieux d'une quotidienneté aujourd'hui perdue, auraient dû nous inciter à nous interroger sur cet « oubli ou déni » de la dernière guerre. Les traces que laissent dans la mémoire les événements extrêmes et récents sont les plus malaisées à repérer, à interpréter. "La mémoire ne cesse de reconstruire sa propre énigme $»^{30}$ et se dérobe à l'investigation du chercheur.

\section{Restituer}

Quand nous étions à Minot, nous n'étions guère conscientes de ces aspects mnémotechniques et réflexifs, tant théoriques que méthodo-logiques. Ainsi, nous ne nous sommes guère interrogées sur les inter-férences introduites sur nos objets de recherche par la situation d'enquête. Nous n'avons pas été suffisamment attentives aux perturbations que l'actualité historique et notre présence pouvaient susciter chez nos interlocuteurs, ni au trouble que nos propres histoires, nos catégories mentales faisaient peser sur nos interrogations ou nos choix d'objets d'études et rejetaient dans le déni des épisodes dont nos interlocuteurs évitaient de parler. La dimension d'implication propre à toute recherche qualitative, désormais incluse dans notre discipline, est absente de nos publications pas plus qu'elle ne figure, sous une forme quelconque, dans nos archives. 
30 Mais il faut comprendre que l'agencement et la morphologie de l'enquête peuvent, en partie, expliquer l'occultation des conditions de production de nos données de terrain. En effet, nous étions le plus fréquemment à deux ou trois sur place et les échanges entre nous étaient constants: nous commentions longuement ce que chacune avait saisi au cours d'une promenade, lors d'une rencontre, d'un entretien, à la lecture d'un document. Les matériaux recueillis, de quelque nature qu'ils soient, étaient sans cesse réfléchis de l'une à l'autre, dans des discussions sans fin. Cette communication intense dans l'agitation affective du "terrain » était davantage pondérée quand nous étions à Paris travaillant toutes quatre au Laboratoire d'anthropologie sociale. Elle donna corps peu à peu à une perspective commune, n'empêchant pas des approches personnelles. Ces façons de procéder, fondées sur l'échange verbal à l'intérieur de l'équipe, la discussion, l'élaboration de textes collectifs ou non, ce souci que nous avions d'éclairer réciproquement l'information ou l'analyse, ont singulièrement coloré cette entreprise. En effet, nous ajustions le cheminement de nos travaux de commentaires constamment relayés de l'une à l'autre, et nous en avons par là même appauvri le journal d'enquête que nous rédigions, à tour de rôle, chaque jour de présence au village. À sa relecture, il apparaît exsangue : il relève plus de l'agenda et, dans sa froide nudité, il ne constitue qu'un aide-mémoire. Cet outil ne fut guère pour nous l'instrument grâce auquel le chercheur tente de mettre à distance ses travaux et à saisir l'impact de ses méthodes d'enquête sur ses informateurs, en d'autres termes, prend conscience du cours de sa recherche. À n'en pas douter, c'est l'interaction à laquelle nous soumettions entre nous les informations recueillies et, surtout, la lecture attentive de l'archive " historique » répertoriée au village qui, nous plongeant dans l'histoire du passé récent de la communauté, nous révélant ce vocabulaire local encore vivant, nous a permis de mieux analyser nos données, de trouver ou de penser la «distance » si nécessaire à la construction de l'objet sur des terrains proches.

31 De même, toutes quatre déterminées à faire aboutir ce travail d'équipe, nous ne nous sommes guère appesanties, du moins par écrit, sur les déroulements personnels de cette longue enquête. On ne trouvera, dans les archives ou dans les publications, aucune trace des arcanes $^{31}$ de la recherche, de nos tâtonnements, de nos doutes ou de nos certitudes, de nos dissensions ou de nos accords; nulle réflexion ici de nos impli-cations diverses. Tout ce qui, sur le terrain ou à Paris, était entre nous de l'ordre de l'émotif ou de l'affectif, nous l'avons passé sous silence. "Minot» est tout entier dans la série d'articles et de livres auxquels il faut ajouter l'exposition photographique qui, au village, a clôturé l'enquête.

32 Nous avons donc cherché avant tout à restituer aux gens de Minot toute la place que méritaient leurs gestes coutumiers, leurs histoires singulières, leurs figures tutélaires. Ce sont eux que nous avons voulu mettre en scène, non le chercheur, et, de cette posture quelque peu " éloignée ", a découlé l'absence de réflexivité, déjà signalée.

C'est seulement après la publication de nos textes que nous nous rendîmes compte de l'importance de cet aspect réflexif entre l'Autre et Soi, non seulement pour la fabrication d'un texte ethnographique dans nos sociétés à écriture et à archives, mais aussi dans une visée de restitution de ces textes aux acteurs locaux.

L'ethnographie sur la « galaxie de Gutenberg » consiste d'abord à se livrer à une réflexion sur les modalités de réception de la parole, puis de transcription d'un discours oral produit dans une société à écriture, et, cela, afin d'élaborer un texte écrit. L'ethnographe passe d'une parole à un texte, il construit un texte qui diffère de la parole entendue puis il 
refait un texte sur un texte. Il opère une mise en correspondance de matériaux hétéroclites; il procède à un entrelacement de réflexions isolées ou de discours prononcés sur divers propos; il met côte à côte des faits actuels ou passés, des signes persistants ou oubliés, des termes vernaculaires toujours employés ou remémorés, et ce, afin de les rendre signifiants, de suggérer récurrences, connexions possibles avec d'autres cultures, avec l'intention d'aboutir à des généralisations. De la mise en réseau de ces éléments locaux, il est attendu de nouvelles façons de comprendre l'Autre et un approfondissement analytique des thèmes étudiés dans le cadre d'une anthropologie sociale.

Mais, pour le lecteur impliqué qui fut notre interlocuteur sur le terrain et dont nous avons emprunté et transcrit la parole, ce mode ethno- graphique de rédaction contient en germes de nombreux problèmes et on peut concevoir les désarrois ou le trouble que pose aux informateurs locaux la restitution de ces textes composés de " collages ", élaborés à partir de « découpages » et constitués de « témoignages » de tous ordres et de tous types.

Ces malentendus surgirent bien évidemment à Minot, où nos articles et nos ouvrages publiés ont été mal reçus. Dans ces divers écrits, nous avions tenté de démonter et de recomposer la trame ignorée et, pour ainsi dire, masquée des rapports sociaux d'une communauté villageoise, en nous appuyant sur une lecture de leurs " papiers » publics ou privés et sur une observation du quotidien vécu par ses membres. Leurs façons de « parler famille ", d'affirmer leurs stratégies matrimoniales et patrimoniales, de vivre et de scander le déroulement de leur vie d'homme et de femme ; les mots utilisés pour dévoiler leur destin, pour entretenir leur réseau social en adéquation avec la culture ordonnancée de leur jardin potager... Des choses ordinaires, en somme, mais qui, révélées publiquement par l'intermédiaire de nos écrits, donnaient, de leur point de vue, une image de personnes "demeurées", voire de "sauvages " $"$ ! Constatations et opinions confortées, corroborées par certains articles de journaux de la presse locale ou nationale qui, non sans malveillance, du moins pour l'un d'entre eux, qui, rendant compte de nos ouvrages, titra sans précaution : «Les Indiens de Bourgogne $»^{33} \ldots$ Mais il y eut plus grave. Pour étayer de façon vivante nos réponses à un entretien que nous avions accordé à un hebdomadaire, la journaliste qui nous avait interrogées se rendit, sans nous prévenir, au village en dépit de nos tentatives réitérées pour l'en dissuader, afin de savoir ce que les gens de Minot pensaient de ce qu'on écrivait sur eux. Elle partit, le livre d'Yvonne Verdier sous le bras et, sur place, elle se fit indiquer les personnes qui avaient servi de modèle à ses trois « héroïnes ». On lui désigna la cuisinière, seule encore en vie et en exercice. La journaliste s'enquit de son nom et la photographia subrepticement. La photographie fut publiée dans le journal avec le véritable nom de la cuisinière - alors qu'Yvonne, comme nous toutes, avait déguisé ${ }^{34}$ tous les patronymes de nos informateurs -, y associant cette phrase tirée du livre : «Elle (la cuisinière Mme... patronyme en clair) ne boit pas, elle ne court pas, elle vole... ». Ainsi mise en cause, la cuisinière de Minot porta plainte contre le journal. Ce dernier se retourna contre les ethnologues. Le tribunal refusa cette façon de se défausser arguant que nous avions fait œuvre scientifique et parlé de ces femmes en termes génériques et que, de plus, en changeant les noms nous n'avions en rien manqué à la déontologie qu'impose tout travail de ce genre ${ }^{35}$. Il donna tort au journal qui dut indemniser la cuisinière. Mais le mal était fait : au village, on pensa que si nous n'avions pas écrit et publié nos ouvrages, rien ne serait arrivé. La brouille était irrémédiable et nous ne sommes jamais retournées à Minot. 
Minot fut - les archives et les textes édités le montrent - un «bricolage scientifique » échafaudé au gré de filiations multiples : l'ethnographie, la littérature, l'art, l'histoire. On le saisit bien, à la lecture du corpus que ces archives offrent. Celui-ci a été constitué à partir d'écrits manuscrits et imprimés anciens ou récents, d'autres ont été collectés oralement, dans l'immédiateté. Même si les données dont il est composé s'enchaînent les unes aux autres, se répondent, elles n'en constituent pas moins un ensemble composite et complexe dont l'usage reste à définir. Toutefois, la lecture que j'ai tenté d'en faire, dans cet ultime regard porté sur "Minot", l'a été pour témoigner de ce qu'on y décèle le moins: la trace, l'empreinte de la volonté résolue de quatre chercheuses à écrire des textes différents tout en puisant leur savoir aux mêmes sources.

\section{NOTES}

1. Nom d'un village qui comptait 370 habitants en 1970, situé au nord-est de la Bourgogne dans le département de la Côte-d'Or, où s'est déroulée, de 1967 à 1975, une étude ethnographique inscrite dans le cadre de la Recherche coopérative sur programme (RCP) du CNRS, consacrée au Châtillonnais. Sur cette dernière, cf. Serge Wolikov, « La RCP du Châtillonnais (1966-1968) ou le rendez-vous interdisciplinaire manqué : la dimension historique d'une revisite ", in Bernard Paillard, Jean-François Simon \& Laurent Le Gall, eds, En France rurale. Les enquêtes interdisciplinaires depuis les années 1960, Rennes, Presses universitaires de Rennes, $2010: 281-300$.

2. Les «papiers » d'Yvonne Verdier, comprenant l'ensemble de ses matériaux de travail sur Minot et ses recherches postérieures sont archivés au Laboratoire d'anthropologie sociale.

3. Cf. Marie-Claude Pingaud \& Françoise Zonabend, « Le fil rouge des femmes ", Ethnologie française, 1991, 21 (4) : 362-365, numéro en hommage à Yvonne Verdier.

4. Tina Jolas, Marie-Claude Pingaud, Yvonne Verdier \& Françoise Zonabend, Une Campagne voisine. Minot, un village bourguignon, Paris, Éd. de la Maison des sciences de l'homme, 1990.

5. «La taille, c'était une planche fendue en deux sur laquelle était inscrit le nom de la famille possédante. Le boulanger gardait une partie, le client l'autre, quand on prenait son pain, on réunissait les deux parties et il faisait une entaille sur le côté par kilo de pain. On payait quand la taille était remplie. On donnait un coup de rabot sur le côté entaillé et on recommençait » (extrait du catalogue de l'exposition photographique «Un village et son terroir, Minot, en Châtillonnais »).

6. Tous ces objets sont désormais déposés au musée de la Vie bourguignonne de Dijon.

7. C'est ainsi que, lors de la seconde édition de La Mémoire longue (Paris, Éd. Jean-Michel Place), en 1999, seules les photographies engrangées au Laboratoire d'anthropologie sociale me servirent d'appui pour cette réédition. J'illustrai en effet la nouvelle couverture par une image très symbolique: celle où l'on voit des habitants de Minot regarder, lors de l'exposition organisée au village, la photographie de leurs aïeuls, prise il $\mathrm{y}$ a quelque quatre-vingts ans, et qui avait servi de couverture à la première édition ! 
L'effet miroir se doublait donc d'un effet gigogne qui répondait à la postface intitulée «Mémoire de la mémoire ». Quant au texte principal, je n'y changeai rien.

8. Il faut remarquer, à notre décharge, que ni le Laboratoire d'anthropologie sociale ni le musée des Arts et Traditions populaires ne nous demandèrent, à la fin de l'enquête, de déposer nos documents de terrain. Je me rappelle même que je voulus verser, dès après leur découverte, à la photothèque du musée les photographies anciennes d'Hyppolite Amiot. La responsable de l'époque me répondit, sans les voir, que cela ne l'intéressait pas, le service possédait déjà trop de clichés de ce type.

9. Chaque document inclus dans un dossier a fait l'objet d'une fiche dont les rubriques étaient : « Type de document - Description matérielle - Contenu intellectuel».

10. Marc Bloch, Les Caractères originaux de l'histoire rurale française, Paris, Armand Colin, 1960.

11. Gaston Roupnel, La Ville et la campagne au XVII e siècle. Étude sur les populations du pays dijonnais, Paris, Armand Colin, 1955.

12. Pierre de Saint-Jacob, Les Paysans de la Bourgogne du Nord au dernier siècle de l'Ancien Régime, Paris, les Belles Lettres, 1960.

13. Georges Potey, Histoire de Minot, A. D. 1 F169 (1-6), Dijon, 1913.

14. Louis-Philippe Chaume, Monographie de la commune de Minot, Aignay-le-Duc, Archives cantonales, 1886.

15. La commune de Minot fut choisie après une brève mission de reconnaissance effectuée dans le Châtillonnais par Isac Chiva et par moi-même, du 27 avril au $1^{\text {er }}$ mai 1967.

16. Ces clichés personnels, nous les leur avions empruntés pour les reproduire. Puis, nous leur rendîmes en plusieurs exemplaires en sorte qu'ils puissent les distribuer autour d'eux. Ainsi ces photographies, auxquelles s'ajoutèrent celles que nous avions prises et les clichés anciens d'Hyppolite Amiot que nous donnâmes à certains, entrèrent dans un système de prêts et de dons propres à entretenir leur patience et l'acceptation de nos multiples entretiens. C'est dire l'importance, pour le bon déroulement de l'enquête, de ces photographies, et ce qui explique peut-être le sort particulier qu'on leur réserva d'emblée : elles furent une sorte de monnaie d'échange qui compensait quelque peu le temps que nos informateurs, si souvent sollicités, passaient à discuter avec nous.

17. À ce propos, on lira l'intervention de Michel Guyonnet, instituteur à Minot de 1967 à 1975, à la journée organisée à la mémoire d'Yvonne Verdier, in Ethnologie française, 1991, $21(4)$.

18. Ce tableau comprend sept rubriques: Minimun et maximun de température Pression atmosphérique - État du ciel - Vie des hommes - Vie des bêtes - Vie des plantes - l'Événement du jour. Présenté à l'exposition photographique, il est, aujourd'hui, déposé au musée de la Vie bourguignonne à Dijon.

19. Cf. Bernard Paillard, Jean-François Simon \& Laurent Le Gall, eds, En France rurale..., op. cit.

20. On nous a, parfois, reproché ce parti pris comme si, ce faisant, nous avions transformé le style de ces témoignages. Nous ne le pensons pas. Simplement, nous les avons traités à la même aune que nous, « écrivains » de leur histoire. 
21. À ce propos, on se reportera à l'article de Gilles Laferté, «Des archives d'enquêtes ethnographiques pour quoi faire ? Les conditions d'une revisite », Genèses, 2006, 63 : 25-45, notamment page 40 .

22. Les quelques réflexions précédentes et suivantes m'ont été suggérées par la relecture $\mathrm{du}$ « dossier de presse », qui contient les notes critiques et comptes rendus suscités par nos diverses publications, et qui figure dans les « archives Minot».

23. Daniel Fabre, «L'ethnologie dans le terroir : à propos du livre Une campagne voisine ", in Libération du jeudi 2 mai 1991.

24. Alain Guerreau, "Ethnologie à Minot: structures et inversion ", Annales. Économies, Sociétés, Civilisation, 1982, 37 (2) : 344-352.

25. Yvonne Verdier, Façons de dire, façons de faire. La Laveuse, la couturière, la cuisinière, Paris, Gallimard, 1979 (« Bibliothèque des sciences humaines ») : 13.

26. «An Anthropology of Women. One of the best things about these books by women about women, and also - up to a point - about men, is that they are overly self-conscious about the fact. They are not defensive. They do not claim to be creating a special, feminine anthropology. It is the coming of age of a type of work that anthropology needs but only as long as it remains integrated within it » (cf. Hervé Varenne, «A New Direction in Community Studies : One French Village, Three Books ", Reviews in Anthropology, 1981, 8 [1] : 105-119). Citons encore: "Notons - sans complaisance - que c'est à des regards féminins que nous devons ce renouvellement de perspectives et [...] ces démarches originales... » (cf. le compte rendu de l'ouvrage de Françoise Zonabend, La Mémoire longue. Temps et histoires au village par Christian Bromberger, in L'Homme, 1981, 21 (2) : 117-120).

27. Florence Weber, «Ethnologues à Minot: quelques questions sur la structure sociale d'un village bourguignon ", Revue française de sociologie, 1981, 22 (2) : 247-262. Cf. aussi : Yvonne Guichard-Claudic, «À la recherche du genre dans l'enquête sur Minot » et Arlette Gautier, "Différences des sexes et division par sexes à Minot», communications au colloque Les Grandes Enquêtes pluridisciplinaires des années $60-70$ : bilans et perspectives, tenu à Brest et à Plozévet, les 16 et 17 mai 2008.

28. Daniel Fabre, «Passeuse aux gués du destin : à propos du livre d'Yvonne Verdier, Façons de dire, Façons de faire... », Critique, 1980, 402 : 1075-1099.

29. «L'absence de toute analyse politique contemporaine est d'autant plus grave qu'elle s'accompagne d'une réduction historique de la politique municipale à une opposition spatiale entre Haut et Bas... » (Florence Weber, « Ethnologues à Minot... ",... art. cit. : 261).

30. Cf. Henri-Pierre Jeudy, « Palinodie », Ethnologie française, 1995, 25 (1) : 65-71.

31. Ainsi, on ne trouvera décrits dans aucun cahier ou dossier, les lieux et leurs décors où nous habitâmes quand nous étions sur le terrain : l'hôtel « louche » à Voulaines (non loin de Minot), puis la pension pour vieilles dames au «château » de Minot où les chambres avaient des parois si minces qu'on entendait toutes les conversations entre les pensionnaires et où, le matin, on était réveillé par le bruit des corbeaux qui venaient, en hiver, becqueter le mastic des fenêtres! Enfin, nous finîmes par prendre pension à l'hôtel-restaurant-café-tabac-distributeur d'essence, situé au centre du village. Il était peu confortable, mais on pouvait travailler dans la salle commune et rencontrer ainsi nombre de personnes. Ce qu'on n'archive pas, c'est le temps passé ensemble, la coprésence, la cospatialité, les réécritures et relectures réciproques, toutes pratiques qui ont donné naissance à nos travaux. 
32. Il me revient en mémoire cette anecdote : lors d'une émission de télévision consacrée à Claude Lévi-Strauss («L'invité du Dimanche » de Pierre Dumayet, du 20 juin 1971, cf. archives Ina), une séquence montrant le travail des ethnologues sur le terrain a été tournée à Minot. Mais, lorsque, devant les caméras, nous avons demandé à nos interlocuteurs de raconter - comme ils l'avaient fait spontanément lors d'entretiens privés - leur expérience d'enfant « au champ les vaches », un grand silence accueillit nos questions. L'idée que la communauté nationale les regardait les rendait muets et nous avons dû changer de sujet.

33. Cf. VSD du 7 au 13 février 1980, n¹27.

34. Tous les noms de nos informateurs ont été déguisés; cependant nous avions gardé le nom du village avec l'autorisation du maire et du conseil municipal.

35. Cf.jugement rendu par la Chambre correctionnelle de la Cour d'appel de Dijon, en février 1981. La revue incriminée était ce même journal VSD, op. cit.

\section{RÉSUMÉS}

Résumé

Quel chercheur en sciences sociales n'a pas été, à un moment de sa carrière, confronté au classement et au devenir des " archives » issues de ses travaux ? C'est une telle aventure qui est relatée dans cet article, à propos des archives issues de l'enquête ethnographique programmée dans le cadre de la RCP du Châtillonnais et menée à Minot, commune du Châtillonnais (Côte-d'Or, France), entre 1967 et 1975, par Tina Jolas, Marie-Claude Pingaud, Yvonne Verdier et Françoise Zonabend. Ce « retour sur archives " par une chercheuse qui les a elle-même produites a permis que se mette en place toute une remémoration sur les modalités de la fabrication de l'enquête ethnographique. Ces archives constituent donc les témoins ultimes du long processus d'élaboration des méthodes et des formes de construction des enquêtes de terrain et de la fabrication des textes publiés. Mais, elles ne peuvent parler ce langage que pour le témoin impliqué que fut l'auteur de l'article et qui les compulse trente ans après.

Abstract

What social scientists have not, at some point in their careers, faced the problem of classifying their work for archives as well as questions about the future of these archives? The story told herein is about the archives of the programmed ethnological survey conducted by the RCP «Châtillonnais » in Minot, a commune in Châtillonnais (Côte-d'Or, France), between 1967 and 1975 by Tina Jolas, Marie-Claude Pingaud, Yvonne Verdier and Françoise Zonabend. This « return to the archives " by a researcher who helped produce them has aroused recollections about the making of this survey. These archives thus attest the long processes of designing the methods and forms of the survey conducted in the field and of the writing of the texts that were published. But they are meaningful only to the parties involved, namely this article's author, who took this archival initiative thirty years later. 
INDEX

Mots-clés : ethnologie de la France, archives, construction du savoir, méthodologie, écriture de l'anthropologie

Keywords : Ethnology of France, Archives, Construction of Knowledge, Methodology, Writing Ethnography

\section{AUTEUR}

\section{FRANÇOISE ZONABEND}

Laboratoire d'anthropologie sociale, Paris 